\title{
European Society of Hypertension International Protocol revision 2010 for the validation of blood pressure measuring devices in adults
}

\author{
Eoin O'Brien ${ }^{a}$, Neil Atkins ${ }^{b}$, George Stergiou ${ }^{c}$, Nikos Karpettas ${ }^{c}$, \\ Gianfranco Parati ${ }^{\text {, }}$ Roland Asmar ${ }^{\mathrm{e}}$, Yutaka Imai ${ }^{\mathrm{f}}$, Jiguang Wang ${ }^{\mathrm{g}}$, \\ Thomas Mengden ${ }^{h}$ and Andrew Shennan; ${ }^{i}$ on behalf of the Working Group \\ on Blood Pressure Monitoring of the European Society of Hypertension
}

Blood Pressure Monitoring 2010, 15:23-38

Keywords: blood pressure, blood pressure measurement, device validation, European Society of Hypertension, International Protocol, protocol

aThe Conway Institute of Biomolecular and Biomedical Research, University College Dublin, Ireland, 'b dabl Ltd., Blackrock Co., Dublin, Ireland, ' ${ }^{\circ}$ ypertension Center, Third University Department of Medicine, Sotiria Hospital, Athens, Greece, distituto Scientifico Ospedale San Luca, IRCCS, Instituto Auxologico Italiano, Milan, Italy, e'Société Française d'Hypertension Artérielle, Filiale de la Société Française de Cardiolgie, Paris, France, ${ }^{\mathrm{f}}$ The Department of Clinical

\section{Introduction}

Measurement of blood pressure is the commonest measurement made in clinical practice, and the interpretation of the figure resulting from that measurement has far-reaching implications for the individual in whom the technique is performed. If the measurement is erroneously low, for example, the patient may be denied the most valuable drug treatment to prevent future stroke and heart attack, whereas if, on the other hand, the measurement is erroneously high, the individual may be commenced on lifelong blood pressure lowering drugs unnecessarily. It is imperative, therefore, that the device being used to measure blood pressure is accurate and, because blood pressure is a complex haemodynamic variable, it is accepted that all blood pressure measuring devices must be validated independently in the clinical setting.

Validation of blood pressure measuring devices began in the 1980s with a series of ad hoc validation protocols on devices [1]. From the 1990s onwards, device validation became more structured with the publication of standards and protocols from the Association for the Advancement of Medical Instrumentation (AAMI) and the British Hypertension Society (BHS) [2-7]. In 2002, the Working Group on Blood Pressure Monitoring of the European Society of Hypertension (ESH), which is composed of experts in blood pressure measurement, many of whom have considerable experience in validating blood pressure measuring devices, published the International Protocol, which simplified previous protocols and
Pharmacology and Therapeutics, Tohoku University Graduate School of Pharmaceutical Science and Medicine, Sendai, Japan, ${ }^{9}$ Centre for Epidemiological Studies and Clinical Trials, Ruijin Hospital, Shanghai Jiaotong University School of Medicine, Shanghai, China, 'University Clinic Bonn, Department of Internal Medicine, Bonn, Germany and 'Guy's and St Thomas' Hospitals, London, UK

Correspondence to Professor Eoin O'Brien, Blood Pressure Unit, St. Michael's Hospital, Dún Laoghaire, Co. Dublin, Ireland

Tel: +35312803865; fax: +35312803688; e-mail: eobrien@iol.ie

Received 23 July 2009 Revised 18 August 2009

Accepted 18 August 2009

was based on evidence from a large number of validation studies [8]. The International Protocol was drafted in such a way as to be applicable to the majority of blood pressure measuring devices on the market. The validation procedure was therefore confined to adults over the age of 30 years (who constitute the majority of subjects with hypertension), and it did not make recommendations for special groups, such as children, pregnant women and the elderly, or for special circumstances, such as during exercise, or for abnormal pathophysiological circumstances, such as atrial fibrillation, or arterial stiffness as may occur in the elderly. The protocol did not preclude investigators and manufacturers from applying the International Protocol to assessment and validation in these circumstances. For full background information on this revision, it is recommended that investigators familiarise themselves with the original protocol, which can be downloaded directly from wwre.dableducational.org.

Initially, the results of validation studies were published in peer-reviewed journals and, every few years, 'state-ofthe-market' papers summarising device accuracy were published in general and specialised journals [9]. However, it became apparent that many of these publications were not accessible to many would-be purchasers of blood pressure measuring devices. To overcome this deficiency, the Working Group of the European Society of Hypertension launched the www.dableducational.org website in 2004. This now receives visits from over 5000 organisations in 100 countries in all continents. 
Since the International Protocol was published in 2002, 78 reported studies have been analysed and this analysis is the evidence base for the changes being incorporated in the first revision of the International Protocol $[1,10]$.

Because of the increasing ban on the use of mercurycontaining sphygmomanometers, there is a need for an equivalent standard device that does not contain mercury.

The following are the basic changes to the revised protocol:

(1) Forms replace free-text results so that all data must be standardised.

(2) The age restriction is reduced from 30 to 25 years to facilitate recruitment.

(3) Phase 1 has been removed, as this is now considered redundant.

(4) As a consequence of improvements in technology, pass levels have been tightened. This is of benefit to manufacturers who strive to produce devices of the highest standard.

(5) Controls on the distribution of observer measurements are introduced to ensure that the intended recruitment ranges are reasonably maintained throughout the full procedure.

(6) Due to difficulties experienced in recruiting subjects in high ranges, the recruitment limits have been relaxed under certain conditions.

Support facilities provided by the dablEducational website to assist validation studies are outlined in Appendix B.

Devices passing the stricter criteria of this revision will be classed as having passed ESH-IP2. It will supersede the original protocol [8] for new studies from 1st July 2010 and for publications from 1st July 2011.

The sphygmocorder [11,12] option described in the previous protocol is not included because no validated model is currently available.

This protocol validation applies only to the recorded measurements and does not extend to average measurements or any other derived statistics.

\section{Validation requirements}

\section{General requirements}

Environment:

(1) The room should be at a comfortable temperature and there should be no noise or other influences that may cause disturbance, such as telephones and pagers.

(2) Ambient noise should not be at a level that could interfere with the auscultation of blood pressures.

Equipment:

(1) Two standard mercury sphygmomanometers, the components of which have been checked carefully before the study, are used as reference standards. They should be within $1 \mathrm{~m}$ of the observers who should be able to follow the menisci at eye level from $40 \mathrm{mmHg}$ to $180 \mathrm{mmHg}$.

(2) Bladders must be available so that, on each subject, there is one of sufficient length to encircle $80 \%-100 \%$ of the arm circumference [13].

(3) Good quality nonelectronic stethoscopes with wellfitting earpieces should be used.

Test device:

(1) The test device bladder should be that provided according to the manufacturer's instructions. If different cuff sizes are recommended by the manufacturer, the appropriate cuff/bladder should be used but no other part of the apparatus should be changed.

(2) If the device detects blood pressure by auscultation, the same microphone(s) must be used throughout the validation test.

(3) If the test device requires software, this should be loaded and tested.

(4) A familiarisation session, involving a number of test measurements, should be performed before starting the validation study. This should be reported.

(5) If the device allows blood pressure measurement by more than one method (e.g. oscillometry and auscultation), then separate validation studies for each measurement method must be performed.

(6) If there is an optional feature intended to assist measurement, such as a facility to mark a blood pressure, then separate validation studies must be performed with and without the facility.

\section{Observer requirements}

A supervisor and two observers are required. The following should be observed:

(1) Observers should have adequate hearing and sight.

(2) Observers should be trained and experienced in blood pressure measurement $[14,15]$.

(3) Observer measurements must be recorded to the nearest $2 \mathrm{mmHg}$.

\section{Subject requirements}

The requirements stated in this protocol refer to a study in the general adult population. Validation studies in specific groups may necessitate modification of these requirements and all such changes or additions should be clearly laid out in Form 1 - Device and Study Details.

Numbers: 33 subjects.

Sex: At least 10 male and 10 female.

Age range: All subjects should be at least 25 years of age Heart rhythm: Sinus rhythm unless the device is being validated for arrhythmias. 
Blood pressure range: 10 to 12 subjects in each of the three SBP and three DBP recruitment ranges as shown in Form 3 - Study Results.

\section{Blood pressure requirements}

(1) Recruitment pressures are intended to ensure a uniform distribution of test pressures across a representative range.

(2) The number of observer test measurements in each pressure range must be between 22 and 44 .

(3) The difference between the range with the highest count and that with the lowest count cannot exceed 19.

(4) The overall SBP range must be from $\leq 100$ to $\geq 170 \mathrm{mmHg}$ and the overall DBP range must be from $\leq 50$ to $\geq 120 \mathrm{mmHg}$.

(5) Ideally recruitment blood pressures should be in the range $90-180 \mathrm{mmHg}$ for SBP and $40-130 \mathrm{mmHg}$ for DBP. However, if patients with blood pressures outside these ranges are available they may be included but only to a maximum of four such pressures.

(6) The number of subjects in each recruitment range must be from 10 to 12 subjects. All SBP pressures below $130 \mathrm{mmHg}$ and DBP pressures below $80 \mathrm{mmHg}$ are counted as 'Low Range'. All SBP pressures above $160 \mathrm{mmHg}$ and DBP pressures above $100 \mathrm{mmHg}$ are counted as 'High Range'.

\section{Accuracy requirements}

The protocol classifies observer-device differences as in Form 3 - Study Results. When comparing and categorising these differences, they are categorised into one of four bands according to their rounded absolute values.

Accuracy is determined by the number differences in these ranges both for individual measurements (Part 1) and for individual subjects (Part 2). To pass, a device must achieve all the minimum Pass Requirements shown.

Accuracy is contingent on strict adherence to the protocol, and results from validations not adhering to this protocol may be called into question.

\section{Validation procedure, analysis and report}

The validation procedure and report should be carried out according to Forms 1-4. These are designed to produce a standard comprehensive and focussed report.

Form 1 - Device and Study Details should be filled at the outset.

A copy of Form 2 - Subject Data is filled for each subject screened. Subject measurements recorded by the observers and, when possible, a test device printout of data recorded for a subject should be attached to each relevant form. Data from these forms should be entered into a computer for appropriate analysis.

The results of the analysis are entered into Form 3 - Study Results.

Form 4 - Study Report describes the report layout which includes all the elements from Form 1 - Device and Study Details and Form 3 - Study Results.

It is imperative that all data are completed so that the report contains all of the information required.

Forms 2 and 3 include numbered clear and shaded boxes. The clear boxes indicate directly recorded data; the shaded ones refer to calculations.

The agreement between the investigator and sponsor should state that a full report of the validation study will be published irrespective of the result.

A photograph of the validated device and cuff against a white background should be provided. 


\section{Form 1 - Device and study details}

\section{Test device details}

Brand Owner Brand

Model

Internal Model Number

Manufacturer

Original Equipment Manufacturer (OEM)

Circle the correct option on each of the following or, if required, complete the explanation beside 'Other'.

$\begin{array}{lllll}\text { Location } & \text { Upper arm } & \text { Wrist } & \text { Finger } & \text { Other } \\ \text { Method } & \text { Oscillometry } & \text { Auscultation } & \text { Doppler } & \text { Other } \\ \text { Purpose } & \text { Clinic measurement } & \text { Self/Home measurement } & \text { ABPM } & \text { Other } \\ \text { Operation } & \text { Automatic } & \text { Semi-automatic } & \text { Manual } & \text { Other }\end{array}$

Automatic: Cuff inflation, deflation and blood pressure determination are fully performed by the device automatically;

Semi-automatic: Blood pressure determination is performed automatically but cuff inflation and/or deflation needs manual operation;

Manual: Blood pressure determination is performed by manually irrespective of inflation and deflation control.

Cuff details including arm circumference ranges (as recommended by the device manufacturer).
Arm cuffs
Small adult:
$\mathrm{cm}$ to
$\mathrm{cm}$
Standard adult:
$\mathrm{cm}$
Large adult:
$\mathrm{cm}$ to
$\mathrm{cm}$
Other:
$\mathrm{cm}$ to
$\mathrm{cm}$
Wrist cuff
$\mathrm{cm}$ to
$\mathrm{cm}$
Wrist Support Method

Other features of the device:

\section{Study details}

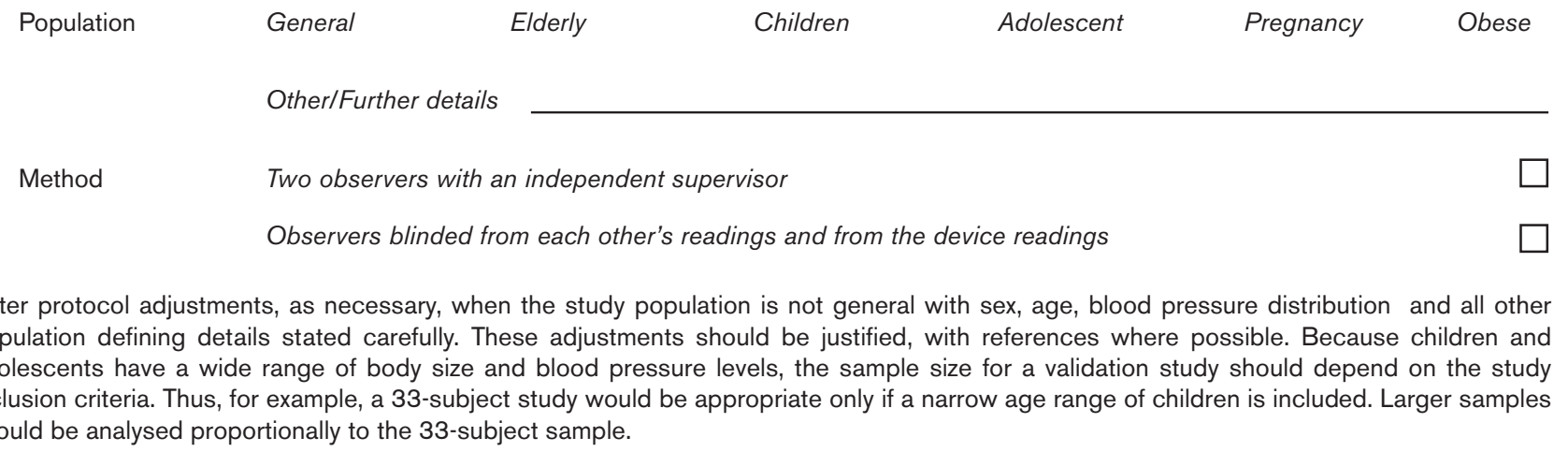
inclusion criteria. Thus, for example, a 33-subject study would be appropriate only if a narrow age range of children is included. Larger samples should be analysed proportionally to the 33-subject sample. 


\section{Form 2 - Subject data}

This form is to be filled by the validation supervisor for each subject. Items in clear boxes are assessed directly. Items in shaded boxes are calculated. All blood pressure measurements are recorded in $\mathrm{mmHg}$ and are carried out sequentially on one arm, whichever is more comfortable for the subject. Including any time required to change cuffs, At least 30 seconds should be allowed between each measurement to avoid venous congestion, but not more than 60 seconds or variability may be increased.

Prerequisites

On a separate sheet, keep a running total of the number of subjects recruited in the Low ( $\mathrm{LL}$ and $\mathrm{L})$, Medium (M), and $\mathrm{High}(\mathrm{H}$ and $\mathrm{HH})$ ranges for SBP (Box 219) and DBP (Box 220).

If any of the following occur during the study, circle the appropriate item in Box 287 and exclude the subject.

- Circle Arrhythmias if the subject is not in sinus rhythm throughout the study.

- Circle Device failure if the device fails to record a measurement after three successive attempts.

- Circle Poor quality sounds if the Korotkov sounds are difficult to auscultate, regardless of the reason.

- Circle Observers disagreement if the observers differ by more than $4 \mathrm{mmHg}$ twice in the same subject.

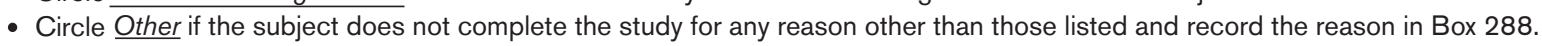

o Starting with the observers, measurements are recorded sequentially alternating between observers and the device in the order BPA, BPB, BP1, BP2, BP3, BP4, BP5, BP6 and BP7.

$\sigma \quad$ Observer measurements should be recorded simultaneously by the observers on separate sheets. They must be checked immediately and, if they differ by more than $4 \mathrm{mmHg}$, they should be taken again. Only enter final measurements on this sheet. Observers must be blinded from each other's measurements and from the device measurements throughout the study.

o If possible, a permanent record of device measurements should be kept. If software is provided, device measurements should be downloaded daily.

\section{Recruitment details}

Date and time

Date of birth

Sex

Arm circumference $\mathrm{cm}$

Wrist circumference $\mathrm{cm}$

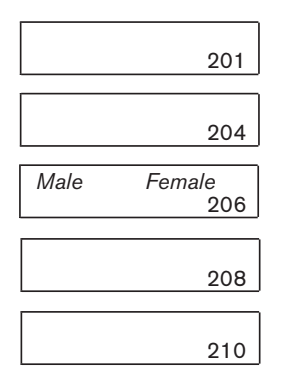

Study number and Subject number

Age

On antihypertensive medication

Cuff for test device

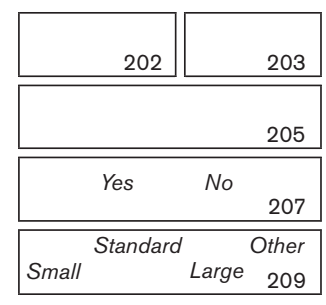

Box 209: This is only required for arm devices. If the arm circumference is such that no suitable cuff is available, circle Cuff size unavailable in Box 287 and exclude the subject.

Box 210: This is only required for wrist devices.

\section{Entry measurements}

Ask the subject to relax for 10-15 min. Then make sure that the subject is seated with legs uncrossed and back supported. Ensure that the arm is supported at heart level. If a wrist device is being tested, the wrist must be supported according to the manufacturer's instructions. If the subject is not seated as described, the posture used must be recorded as a protocol adjustment.

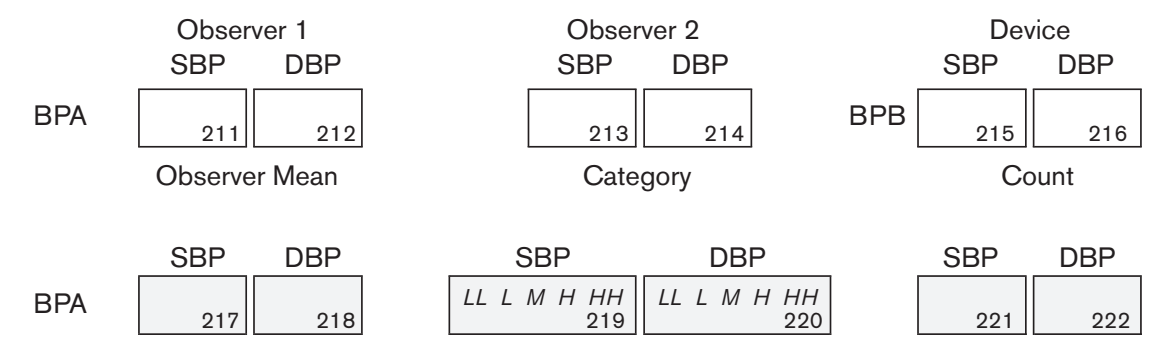

Boxes 217, 218: Enter the rounded up averages of Boxes 211 and 213 and of Boxes 212 and 214 respectively.

Box 219: $\quad$ Circle the range according to the BP in Box 217. LL: <90, L: 90-129, M: 130-160, H: 161-180, HH: >180.

Box 220: $\quad$ Circle the range according to the BP in Box 218. LL: <40, L: 40-79, M: 80-100, H: 101-130, HH: >130.

Boxes 221, 222: Update the running totals, for the ranges (counting $L L$ and $L$ together and counting $\mathrm{HH}$ and $\mathrm{H}$ together) in Boxes 219 and 220 , and enter them respectively.

- If both Box 221 and Box 222 are greater than 12 or if an $L L$ or $H H$ is circled and there are already 4 such items circled, circle Ranges complete in Box 287 and exclude the subject.

- If one of Box 221 or Box 222 is greater than 12 but the other is not, it may be necessary to include this subject, and remove another, in order to achieve the correct range distribution; in the removed subject, circle Range adjustment in Box 287. 


\section{Validation measurements}

\begin{tabular}{|c|c|c|c|c|c|c|c|c|}
\hline & & $P 1$ & $\mathrm{~B}$ & 3 & & $P 5$ & & \\
\hline & SBP & DBP & SBP & DBP & SBP & DBP & SBP & DBP \\
\hline Observer 1 & 223 & 224 & 225 & 226 & 227 & 228 & 229 & 230 \\
\hline Observer 2 & 231 & 232 & 233 & 234 & 235 & 236 & 237 & 238 \\
\hline Observer mean & 239 & 240 & 241 & 242 & 243 & 244 & 245 & 246 \\
\hline $\begin{array}{l}\text { Observer } \\
\text { difference }\end{array}$ & 247 & 248 & 249 & 250 & 251 & 252 & 253 & 254 \\
\hline & & $\mathrm{P} 2$ & & P4 & & P6 & & \\
\hline & SBP & DBP & SBP & DBP & SBP & $\mathrm{DBP}$ & Diffe & ce \\
\hline Device & 255 & 256 & 257 & 258 & 259 & 260 & $\begin{array}{l}0-5 \\
6-10\end{array}$ & $\begin{array}{l}\mathrm{nHg} \\
\mathrm{mHg}\end{array}$ \\
\hline $\begin{array}{l}\text { Device-Previous } \\
\text { observer }\end{array}$ & 261 & 262 & 263 & 264 & 265 & 266 & $\begin{array}{l}11- \\
>15\end{array}$ & $\begin{array}{l}\mathrm{mmHg} \\
\mathrm{mHg}\end{array}$ \\
\hline $\begin{array}{l}\text { Device-Next } \\
\text { observer }\end{array}$ & 267 & 268 & 269 & 270 & 271 & 272 & $\begin{array}{l}\text { Within } \\
\text { SBP }\end{array}$ & $\begin{array}{r}\mathrm{nmHg} \\
\mathrm{DBP}\end{array}$ \\
\hline Error group & $\begin{array}{|rrrr|}A & B & C & D \\
& 273 \\
\end{array}$ & $\begin{array}{|rrrr|}A & B & C & D \\
& & 274 \\
\end{array}$ & $\begin{array}{|rrrr|}A & B & C & D \\
& & 275 \\
\end{array}$ & $\begin{array}{|rrrr|}A & B & C & D \\
& 276 \\
\end{array}$ & $\begin{array}{|llll|}A & B & C & D \\
& & 277 \\
\end{array}$ & $\begin{array}{rrr}A & B & C \\
& 278 \\
\end{array}$ & 279 & 280 \\
\hline Observer value & 281 & 282 & 283 & 284 & 285 & 286 & & \\
\hline
\end{tabular}

Boxes 239-246: Enter the rounded up averages of Boxes 223 and 231, of Boxes 224 and 232, of Boxes 225 and 233 , of Boxes 226 and 234, of Boxes 227 and 235, of Boxes 228 and 236, of Boxes 229 and 237 and of Boxes 230 and 238 respectively.

Boxes 247-254: Enter the differences Box 223-Box 231, Box 224-Box 232, Box 225-Box 233, Box 226-Box 234, Box 227-Box 235, Box 228-Box 236, Box 229-Box 237, and Box 230-Box 238 respectively.

Boxes 261-266: Enter the 'Device-Previous Observer Mean' differences. These are Box 255-Box 239, Box 256-Box 240, Box 257-Box 241, Box 258-Box 242, Box 259-Box 243, and Box 260-Box 244 respectively.

Boxes 267-272: Enter the 'Device-Next Observer Mean' differences. These are Box 255-Box 241, Box 256-Box 242, Box 257-Box 243, Box 258-Box 244, Box 259-Box 245, and Box 260-Box 246 respectively.

$\sigma \quad$ Each device measurement is compared to the nearer of the previous and next observer measurement. As described below, the smaller error is circled and the corresponding observer measurement is copied to aid the analysis.

Box 273: $\quad$ First, circle the smaller value, in absolute terms, of Boxes 261 and 267 (Box 261 if both are the same).

Then circle $A$ if this is less than or equal to $5, B$ if this is $6-10, C$ if this is $11-15$ or $D$ if this is greater than 15 .

Boxes 274-278: Circle A, B, C or D, as described for Box 273, based on the smallest absolute values (also circled) of Boxes 262 and 268, Boxes 263 and 269, Boxes 264 and 270, Boxes 265 and 271 and Boxes 266 and 272 respectively.

Box 279: $\quad$ Enter the number of 'A's circled in Boxes 273, 275 and 277.

Box 280: $\quad$ Enter the number of 'A's circled in Boxes 274, 276 and 278.

o The numbers of 'A's, 'B's and 'C's will be used in the analysis to calculate the number of device-observer differences within $5 \mathrm{mmHg}$, $10 \mathrm{mmHg}$ and $15 \mathrm{mmHg}$.

Box 281: $\quad$ Enter the value in Box 239 if Box 261 is circled or Box 241 if Box 267 is circled.

Box 282: $\quad$ Enter the value in Box 240 if Box 262 is circled or Box 242 if Box 268 is circled.

Box 283: $\quad$ Enter the value in Box 241 if Box 263 is circled or Box 243 if Box 269 is circled.

Box 284: $\quad$ Enter the value in Box 242 if Box 264 is circled or Box 244 if Box 270 is circled.

Box 285: $\quad$ Enter the value in Box 243 if Box 265 is circled or Box 245 if Box 271 is circled.

Box 286: $\quad$ Enter the value in Box 244 if Box 266 is circled or Box 246 if Box 272 is circled.

\section{Signoff}

Please use the comments to explain unlisted subject exclusion or any problems during the procedure

\begin{tabular}{l|lllll|}
\hline Excluded & $\begin{array}{l}\text { Ranges complete } \\
\text { Poor quality sounds }\end{array}$ & $\begin{array}{l}\text { Range adjustment } \\
\text { Cuff size unavailable }\end{array}$ & $\begin{array}{l}\text { Arrhythmias } \\
\text { Observer disagreement }\end{array}$ & $\begin{array}{l}\text { Device failure } \\
\text { Distribution }\end{array}$ & Other \\
\hline & & & & 287 \\
\hline
\end{tabular}

Note: Subjects initially included may be excluded due to changes in pressure. See observer measurements in each recruitment range in Form 3 - Study Results.

If a printout of test device data is possible, print the device data for this subject and attach it to this form. 


\section{Form 3 - Study results}

The data from Form 2 - Subject Data for each subject should be appropriately analysed so that the results on this form can be completed. All references to boxes 201-289 refer to values obtained from all of the Forms 2 from the relevant subjects. All blood pressure measurements are in mmHg.

Table 1 Screening and recruitment details

Screening and recruitment

Total screened

Total excluded

Ranges complete

Range adjustment

Arrhythmias

Device failure

Poor quality sounds

Cuff size unavailable

Observer disagreement

Distribution

Other reasons

Total recruited

*Explanation summary
Recruitment ranges
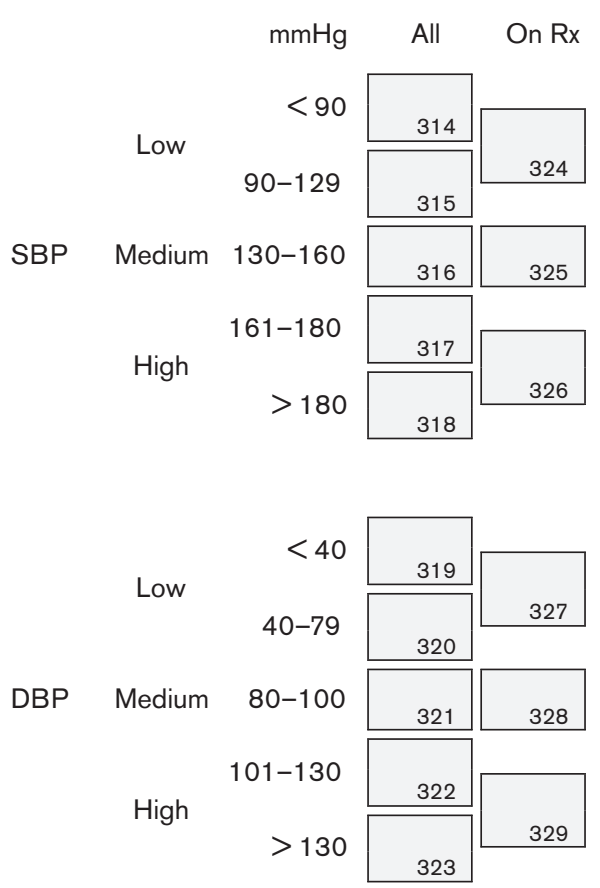

Box 301: $\quad$ The total number of subjects screened, regardless of whether or not they were included in the study.

Box 302: $\quad$ The total number excluded. This equals the sum of Boxes 303-311.

Box 303: $\quad$ The number of subjects excluded with Ranges complete circled in Box 287 (Form 2 for each excluded subject).

Box 304: $\quad$ The number of subjects excluded with Range adjustment circled in Box 287.

Box 305: $\quad$ The number of subjects excluded with Arrhythmias circled in Box 287.

Box 306: $\quad$ The number of subjects excluded with Device failure circled in Box 287.

Box 307: $\quad$ The number of subjects excluded with Poor quality sounds circled in Box 287.

Box 308: $\quad$ The number of subjects excluded with Cuff size availability circled in Box 287.

Box 309: $\quad$ The number of subjects excluded with Observer disagreement circled in Box 287.

Box 310: $\quad$ The number of subjects excluded with Distribution circled in Box 287.

Box 311: The number of subjects excluded with Other reasons circled in Box 287. A summary of those reasons must be provided in Box 313 .

Box 312: $\quad$ The total recruited equals the number screened (Box 301) less the number excluded (Box 302). This should equal 33 except in validations in some specific populations.

Box 313: $\quad$ A summary of why those counted in Box 311 were excluded (Box 288).

Boxes 314-323: In a completed study in a general adult population, the sum of Boxes 314 and 315, Box 316, the sum of Boxes 317 and 318 , the sum of Boxes 319 and 320 , Box 321 and the sum of Boxes 322 and 323 must each be between 10 and 12. The sum of Boxes $314,318,319$ and 323 must be at most 4. The sum of Boxes 314-318 and the sum of Boxes 319-323 must each be exactly 33. Studies in specific populations may have different restrictions and totals (Boxes 219 and $220-$ Form 2 for each included subject).

Boxes 324-329: The number of subjects in each range on antihypertensive medication (Boxes 207, 219 and 220). 


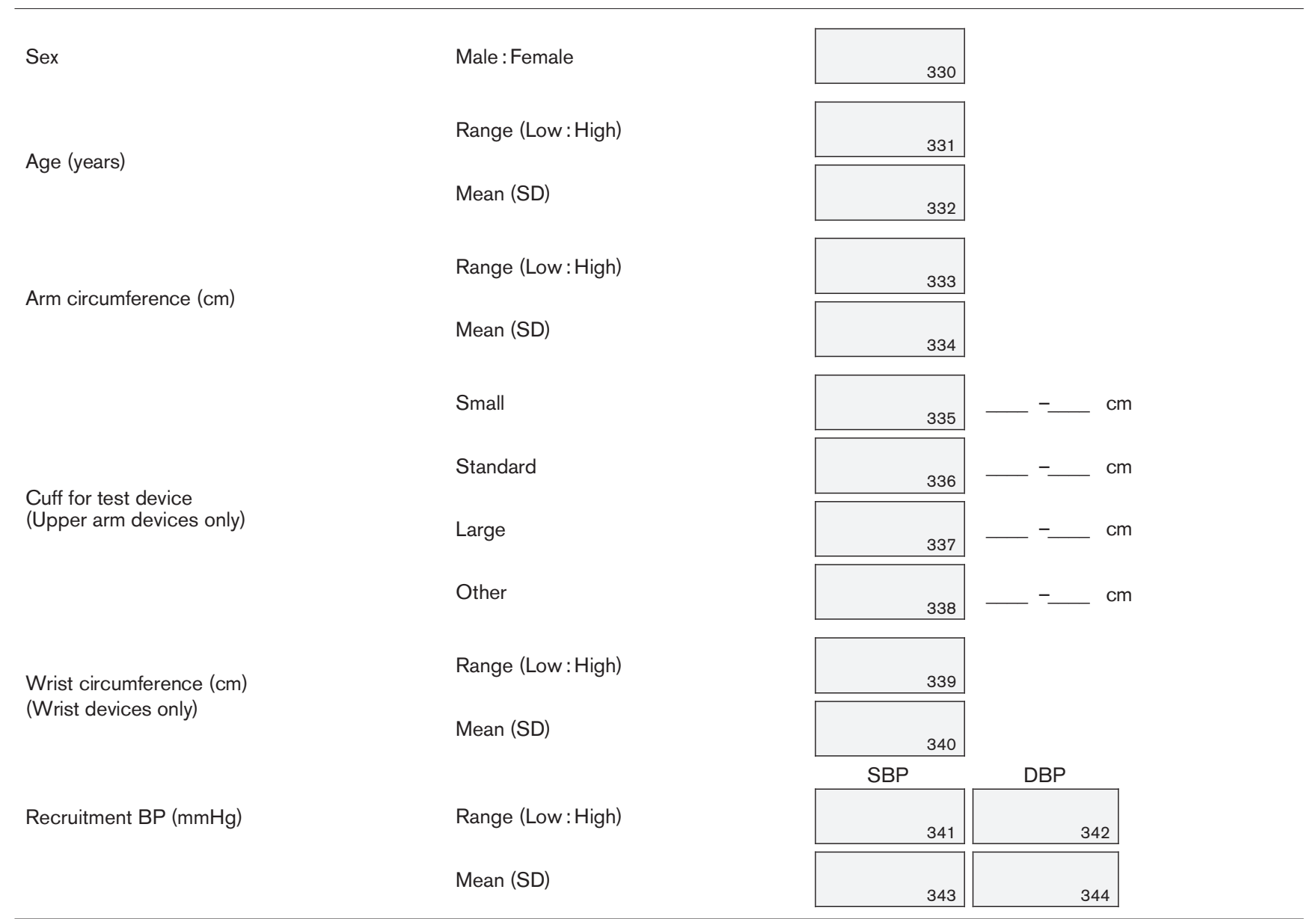

Note: The values in Boxes 314-380 refer only to the final recruited subjects, each of whom contributes SBP and DBP measurements for analysis. Excluded subjects are not included in any of this analysis.

Bох 330: $\quad$ Enter the number of males, a colon and the number of females. They should total 33 except in validations in some specific populations. If the minimum requirements ( 10 for a general population) are not met, subjects must be replaced as necessary (Box 206).

Box 331: Enter the age of the youngest subject, a colon and the age of the oldest subject e.g. 31:74. Subjects outside the required range (25 and over for a general population) are not permitted (Box 205).

Box 332: $\quad$ Enter the mean and, in parentheses, the SD of the subject ages. Values should be rounded up to one decimal place e.g. 52.3 (11.9) (Box 205).

Bох 333: $\quad$ Enter the smallest arm circumference, a colon and the largest arm circumference e.g. 24:34 (Box 208).

Box 334: $\quad$ Enter the mean and, in parentheses, the SD of the subject arm circumferences. Values should be rounded up to one decimal place e.g. 29.0 (3.1) (Box 208).

Box 335: If a small cuff was supplied, enter the number of subjects on whom it was used. If it was not supplied, enter an ' $X$ '. Enter the arm sizes for which it is recommended beside it. (Applicable only for arm devices) (Box 209).

Bох 336: $\quad$ Enter the number of subjects on whom a standard (or medium) cuff was used. Enter the arm sizes for which it is recommended beside it. (Applicable only for arm devices) (Box 209).

Box 337: If a large cuff was supplied, enter the number of subjects on whom it was used. If it was not supplied, enter an ' $X$ '. Enter the arm sizes for which it is recommended beside it. (Applicable only for arm devices) (Box 209).

Box 338: If a different size cuff was supplied, enter the number of subjects in whom it was used. If no such cuff was supplied, enter an ' $X$ '. Enter the arm sizes for which it is recommended beside it. (Applicable only for arm devices) (Box 209).

Box 339: $\quad$ Enter the smallest wrist circumference, a colon and the largest wrist circumference e.g. 15:22 (Applicable only for wrist devices) (Box 210).

Box 340: $\quad$ Enter the mean and, in parentheses, the SD of the subject wrist circumferences. Values should be rounded up to one decimal place e.g.18.1(2.3) (Applicable only for wrist devices) (Box 210).

Boxes 341-342: Enter the lowest pressure, a colon and the highest pressure from BPA measurements only e.g.104:180. (Boxes 217 and 218).

Boxes 343-344: Enter the mean and, in parentheses, the SD of the subject pressures from BPA measurements only. Values should be rounded up to one decimal place e.g. 140.4 (20.3). (Boxes 217 and 218). 


\section{SBP}

Overall range (Low : High)

Low $(<130)$

Medium (130-160)

High (>160)

Maximum difference
DBP

Overall range (Low: High)

Low $(<80)$

Medium (80-100)

High (>100)

Maximum difference

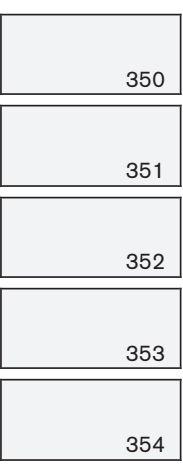

This section analyses the distribution of comparative measurements.

Box 345: $\quad$ Enter the lowest pressure, a colon and the highest SBP from the observer measurements (Boxes 281, 283 and 285).

Boxes 346-348: The observer measurements (three per subject) for SBP are categorised similarly to the recruitment ranges. Enter the counts of measurements falling into each range. These must total 99 (Boxes 281, 283 and 285).

Box 349: $\quad$ Subtract the smallest value from Boxes 346 to 348 from the largest one and enter the result.

Box 350: $\quad$ Enter the lowest pressure, a colon and the highest DBP from the observer measurements (Boxes 282, 284 and 286).

Boxes 351-353: The observer measurements (three per subject) for DBP are categorised similarly to the recruitment ranges. Enter the counts of measurements falling into each range. These must total 99 (Boxes 282, 284 and 286).

Box 354: $\quad$ Subtract the smallest value from Boxes 351 to 353 from the largest one and enter the result.

o. In order to ensure a uniform distribution, there must be at least 22 measurements and at most 44 measurements (Boxes 346 to 348 and 351 to 353) in each of the low, medium and high ranges and the maximum differences (Boxes 349 and 354) must be at most 19. If not, further recruitment will be necessary. Subjects to be excluded will be those whose pressures drifted from recruitment pressures.

- The overall SBP range must be from $\leq 100 \mathrm{mmHg}$ to $\geq 170 \mathrm{mmHg}$ and the overall DBP range must be from $\leq 50 \mathrm{mmHg}$ to $\geq 120 \mathrm{mmHg}$. If not, further recruitment will be necessary. Subjects to be excluded will be the last recruited within the relevant ranges.

o The minimum number of replacements should take place. If a subject is replaced for either of these reasons, circle Distribution in Box 287 of Form 2 for that subject.

$\sigma \quad$ In validations carried out in specific populations requiring more than 33 subjects but with similar blood pressure distributions, similar proportions should be used. If the blood pressure distribution in the specific population differs from the standard distribution, ignore this table but comment on the distribution in the discussion.

\section{Table 4 Observer differences}

This section is for the differences in pressures between the two observers

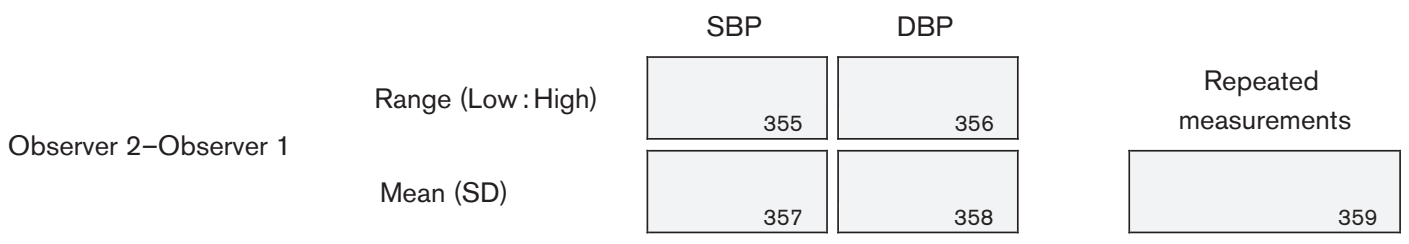

Boxes 355-356: Enter the lowest difference, a colon and the highest difference between the observers. Include the signs e.g. $-3:+4$. (Boxes 247, 249, 251 and 253 and Boxes 248, 250, 252 and 254). If the range is outside $-4:+4$, then this is a violation. Relevant subjects should be excluded, by reason of Observer Disagreement, and replaced.

Boxes 357-358: Enter the mean and, in parentheses, the SD of the observer differences. Values should be rounded up to one decimal place e.g. 0.3 (1.2) (Boxes 247, 249, 251 and 253 and Boxes 248, 250, 252 and 254).

Boxes 359: $\quad$ Enter the number of measurements that were repeated in the included subjects because observers were more than $4 \mathrm{mmHg}$ apart. 
Table 5 Validation results

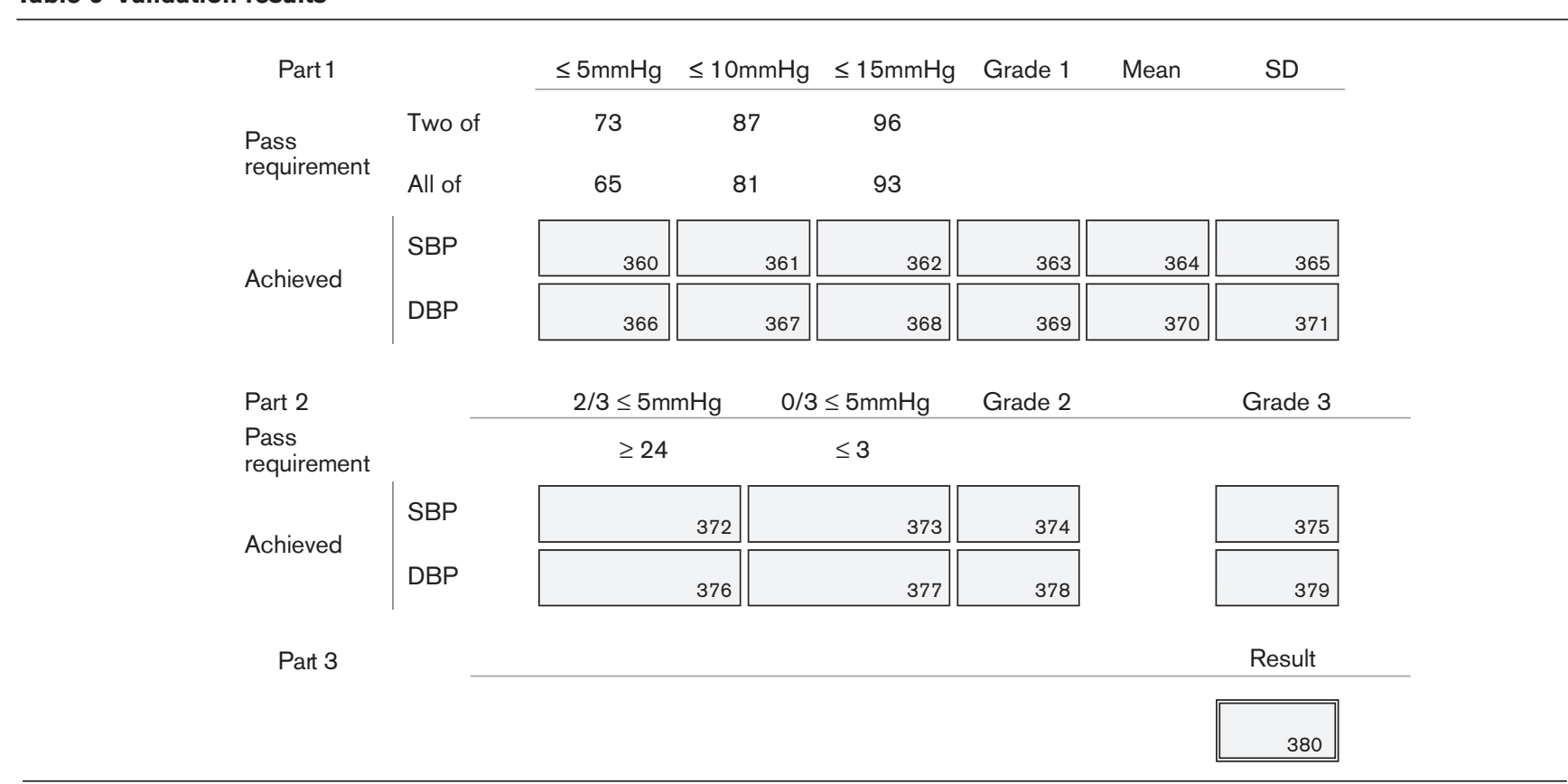

$\sigma \quad$ In order for the device to pass, all protocol requirements must be fulfilled. A fail in any part will result in an overall fail

Box 360: $\quad$ Enter the number of SBP differences (at most 99) between observer and device measurements falling within $5 \mathrm{mmHg}$. (The total number of Boxes 273, 275 and 277 circled $A$ in the 33 subjects)

Box 361: $\quad$ Enter the number of SBP differences (at most 99) between observer and device measurements falling within $10 \mathrm{mmHg}$. (The total number of Boxes 273, 275 and 277 circled A or B in the 33 subjects)

Box 362: $\quad$ Enter the number of SBP differences (at most 99) between observer and device measurements falling within $15 \mathrm{mmHg}$. (The total number of Boxes 273, 275 and 277 circled A, B or C in the 33 subjects)

Box 363: If Boxes 360, 361 and 362 fulfil the Pass requirements, then this is 'Pass'; otherwise, it is 'Fail'.

Boxes 364-365: Enter the mean and standard deviation respectively of the 99 SBP differences between observer and device measurements. (Use data from circled Boxes 261 or 267, 263 or 269 and 265 or 271)

Box 366: $\quad$ Enter the number of DBP differences (at most 99) between observer and device measurements falling within $5 \mathrm{mmHg}$. (The total number of Boxes 274, 276 and 278 circled $A$ in the 33 subjects)

Box 367: $\quad$ Enter the number of DBP differences (at most 99) between observer and device measurements falling within $10 \mathrm{mmHg}$. (The total number of Boxes 274, 276 and 278 circled A or B in the 33 subjects)

Box 368: $\quad$ Enter the number of DBP differences (at most 99 ) between observer and device measurements falling within $15 \mathrm{mmHg}$. (The total number of Boxes 274, 276 and 278 circled A, B or $\mathrm{C}$ in the 33 subjects)

Box 369: If Boxes 366, 367 and 368 fulfil the Pass requirements, then this is 'Pass'; otherwise, it is 'Fail'.

Boxes 370-371: Enter the mean and standard deviation respectively of the 99 DBP differences between observer and device measurements. (Use data from circled Boxes 262 or 268, 264 or 270 and 266 or 272)

Box 372: $\quad$ Enter the number of subjects (at most 33) with two or three of the absolute differences between observer and device SBP measurements within $5 \mathrm{mmHg}$. (Box 279 is 2 or 3 )

Box 373: $\quad$ Enter the number of subjects (at most 33) with none of the absolute differences between observer and device SBP measurements within $5 \mathrm{mmHg}$. (Box 279 is 0 )

Box 374: If Boxes 372 and 373 fulfil the Pass requirements, then this is 'Pass'; otherwise, it is 'Fail'.

Box 375: If Boxes 363 and 374 are both 'Pass', then this is 'Pass'; otherwise, it is 'Fail'.

Box 376: $\quad$ Enter the number of subjects (at most 33) with two or three of the absolute differences between observer and device DBP measurements within $5 \mathrm{mmHg}$. (Box 280 is 2 or 3)

Box 377: $\quad$ Enter the number of subjects (at most 33) with none of the absolute differences between observer and device DBP measurements within $5 \mathrm{mmHg}$. (Box 280 is 0 )

Box 378: If Boxes 376 and 377 fulfil the Pass requirements, then this is 'Pass'; otherwise, it is 'Fail'.

Box 379: If Boxes 369 and 378 are both 'Pass', then this is 'Pass'; otherwise, it is 'Fail'.

Box 380: If Boxes 375 and 379 are both 'Pass', then this is 'Pass'; otherwise, it is 'Fail'.

o. In validations carried out in specific populations requiring more than 33 subjects, proportionally equivalent passing criteria should be used. 


\section{Form 4 - Study report}

Each device should be reported separately, even if more than one device is validated in the same study.

\section{Title}

It is important that the title conveys the nature of the validation both concisely and comprehensively.

The title should read Validation CIRCUMSTANCE of the MANUFACTURER MODEL TYPE blood pressure monitor USE according to the European Society of Hypertension International Protocol revision 2010.

Where MANUFACTURER is the name of the manufacturer or brand.

MODEL is the model number. If more than one number is used, the alternatives, including internal model numbers, should follow in parentheses.

TYPE describes the nature of the monitor. This is typically upper arm, wrist or $A B P M$. If it is not an automatic monitor, it should also include an appropriate adjective such as manual, semi-automatic or hybrid. USE is optional but may supplement TYPE to describe the monitor's intended use. It could be general, for example upper arm monitors might be primarily intended 'for self measurement' or 'for clinic use', or specific, for example 'for clinic use in low resource settings'. Commas should be added for clarity.

CIRCUMSTANCE is required only if the study is not carried out in a general population or where the subjects are not at rest. It should state the population, group or other factor that defines the applicability of the results. Commas should be added for clarity.

Two examples are shown below and a third is shown in Appendix A.

Validation of the Gizmo ABC-01 ABPM blood pressure monitor according to the European Society of Hypertension International Protocol revision 2010.

Validation, in the elderly, of the Gizmo ABC-02 upper arm blood pressure monitor, for self measurement, according to the European Society of Hypertension International Protocol revision 2010.

\section{Device details}

This is taken from the Device details section of Form 1 - Device and Study Details. A digital photograph of the monitor used in the study should be included. Do not use a photograph, of a similar model, downloaded from the web or supplied by the manufacturers.

\section{Methodology}

This is described in two paragraphs and it contains the information in the Study details section of Form 1 - Device and Study Details.

\section{Familiarisation}

A brief description of the familiarisation session should be provided. Any difficulties should be reported.

\section{Recruitment}

The population should be outlined and the method of selecting the sample should be described. Difficulties in recruitment should be described and how they were overcome.

\section{Procedure}

Outline any adjustment to the protocol due to validation in a nongeneral population, or otherwise, and outline any other exceptional issues relating to the study. If the protocol was followed as written, this should be stated as follows: The European Society of Hypertension International Protocol revision 2010 for the validation of blood pressure measuring devices in adults was followed precisely. The check boxes the Study Details section of Form 1 - Device and Study Details should both be ticked. If so, a sentence should state this as follows: Overseen by an independent supervisor, measurements were recorded by two observers blinded from both each other's readings and from the device readings. If not, it is likely to constitute a serious violation and needs to be explained.

Problems encountered (apart from recruitment issues), including device faults, should be described along with their resolutions. All problems should be put in the context of the stage in the validation: This would give the total number of subjects completed at that time e.g. the device fell onto a concrete floor after the 25th subject was completed. It was recalibrated and found not to have been damaged.

\section{Results}

The results will comprise the tables in Form 3 - Study Results along with a graphical presentation. No text should be added.

\section{Plots}

These are mean-difference plots [16,17] and they should be exactly as shown in the example plot (Appendix A). The $x$-axis of these plots represents blood pressures in the systolic range $80 \mathrm{mmHg}-190 \mathrm{mmHg}$ and the diastolic 
range $30 \mathrm{mmHg}-140 \mathrm{mmHg}$. The $y$-axes represent errors from $-30 \mathrm{mmHg}$ to $+30 \mathrm{mmHg}$. Horizontal reference lines are drawn at $5 \mathrm{mmHg}$ intervals from $+15 \mathrm{mmHg}$ to $-15 \mathrm{mmHg}$. The mean of each device pressure and its corresponding observer pressure is plotted against their difference with a point. Differences greater than $30 \mathrm{mmHg}$ are plotted at $30 \mathrm{mmHg}$. Differences less than $-30 \mathrm{mmHg}$ are plotted at $-30 \mathrm{mmHg}$. The same scales should be used for both SBP and DBP plots.

Where points are superimposed, these should be indicated either by proportionately larger points or by different symbols.

\section{Discussion}

The possible effect of any problems encountered should be raised - even if this is to state that there was no effect. A brief comment should be given on the sample and, with reference to the plots, the distribution of pressures. This should include an account of how well the population is represented. If this is poor (for example, if the pressures in a blood pressure range are clustered within that range), an explanation of why a better sample could not be obtained must be provided along with justification for the applicability of the results. Where a special population is used, any adjustments not defined by the population should be justified. If Korotkov Phase IV was used, the effect of this on the accuracy of DBP should be discussed. If previously published validation studies exist for this device, their results should be briefly compared and contrasted to those of the current study.

\section{Conclusion}

The conclusion as to whether the device is accurate for use in the population should be stated. If the results are particularly sensitive to correct use (e.g. most wrist devices) then this caution must be stated.

\section{Acknowledgements and conflicts of interest}

All acknowledgements and any conflict of interest should be provided as appropriate. These include any sources of funding or provision of equipment. The manner by which the test devices were acquired should be stated.

\section{Reference}

The paper should be referenced. If there are previous validation studies on this device, they should be referenced.

\section{References}

1 O'Brien E, Atkins N. Validation and reliability of blood pressure monitors. In: White W, editor. Blood pressure monitoring in cardiovascular medicine and therapeutics. US: Humana Press Inc; 2007, pp. 97-132.

2 Association for the Advancement of Medical Instrumentation. The National Standard of Electronic or Automated Sphygmomanometers. Arlington, Virginia: AAMI; 1987.

3 O'Brien E, Petrie J, Littler W, de Swiet M, Padfield PL, O'Malley K, et al. The British Hypertension Society Protocol for the evaluation of automated and semi-automated blood pressure measuring devices with special reference to ambulatory systems. J Hypertens 1990; 8:607-619.

4 O'Brien E, Petrie J, Littler WA, de Swiet M, Padfield PL, Altman D, et al. The British Hypertension Society Protocol for the evaluation of blood pressure measuring devices. J Hypertens 1993; 11 (Suppl 2):S43-S63.

5 American National Standard. Electronic or automated sphygmomanometers. Association for the Advancement of Medical Instrumentation. 3330 Washington Boulevard, Suite 400, Arlington, VA 22201-4598. 1993.

6 American National Standard. Electronic for manual, electronic, or automated sphygmomanometers. Association for the Advancement of Medical Instrumentation. 1110 N. Glebe Road, Suite 220 Arlington, VA 22201-4795. 2003.

7 American National Standard. Non-invasive sphygmomanometers - Part 2: Clinical validation of automated measurement type. ANSI/AAMI/ISO 81060-2:2009. Association for the Advancement of Medical Instrumentation, Arlington, Virginia: AAMI; 2009.

8 O'Brien E, Pickering T, Asmar R, Myers M, Parati G, Staessen J, et al.; on behalf of the Working Group on Blood Pressure Monitoring of the European Society of Hypertension. International protocol for validation of blood pressure measuring devices in adults. Blood Press Monit 2002; 7:3-17.
9 O'Brien E, Waeber B, Parati G, Staessen G, Myers MG; on behalf of the European Society of Hypertension Working Group on Blood Pressure Monitoring. Blood pressure measuring devices: validated instruments. $B M J$ 2001; 322:531-536.

10 Stergiou G, Karpettas N, Atkins N, O'Brien E. European Society of Hypertension International Protocol for the validation of blood pressure monitors: a critical review of its application and rationale for revision. Blood Press Monit 2010; 15:39-48.

11 O'Brien E, Atkins N, Mee F, Coyle D, Syed S. A new audio-visual technique for recording blood pressure in research: the Sphygmocorder. J Hypertens 1995; 13:1734-1737.

12 Atkins N, O'Brien E, Wesseling KH, Guelen I. Increasing observer objectivity with audio-visual technology: the Sphygmocorder. Blood Press Monit 1997; 2:269-272.

13 Forsberg SA, DE Guzman M, Berlind S. Validity of blood pressure measurement with cuff in the arm and forearm. Acta Med Scand 1979; 188:389-396.

14 O'Brien E, Mee F, Atkins N, O'Malley K, Tan S. Training and assessment of observers for blood pressure measurement in hypertension research. J Human Hypertension 1991; 5:7-10.

15 Mee F, Atkins N, O'Brien E. Problems associated with observer training in blood pressure measurement. J Human Hypertens 1994; 8:293.

16 Tukey JW. Exploratory data analysis. Reading Massachusetts: AddisonWesley; 1977.

17 Bland JM, Altman DG. Statistical methods for assessing agreement between two methods of clinical measurement. Lancet 1986; i:307-310.

18 Atkins N, O'Brien E. The dablEducational Trust device equivalence procedure. Blood Press Monit 2007; 12:245-249.

19 Omboni S, Costantini C, Pini C, Bulegato R, Manfellotto D, Rizzoni D, et al. PA.NET International quality certification protocol for blood pressure monitors. Blood Press Monit 2008; 13:285-289. 


\section{Appendices}

\section{Appendix A: Example of a completed validation report}

Validation of the Acme A1 upper arm blood pressure monitor, for clinic use and self measurement, according to the European Society of Hypertension International Protocol revision 2010.

\section{Device details}

$\begin{array}{ll}\text { Brand } & \text { Acme Model } \\ \text { Manufacturer } & \text { Acme } \\ \text { Location } & \text { Upper arm } \\ \text { Method } & \text { Oscillometry } \\ \text { Purpose } & \text { Clinic and self measurement } \\ \text { Operation } & \text { Automatic } \\ \text { Arm cuffs } & \text { Standard adult: } 20 \mathrm{~cm} \text { to } 32 \mathrm{~cm} \text { and Large adult: } 33 \mathrm{~cm} \text { to } 43 \mathrm{~cm} \\ \text { Other features } & \text { Facility for averaging the last three measurements }\end{array}$

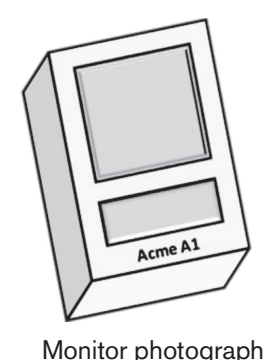

\section{Methodology}

\section{Familiarisation}

Twelve test-measurements were carried out. No problems were encountered.

\section{Recruitment}

Hypertensive subjects were recruited from those attending the hypertension clinic in the ABC Hospital. Some participated immediately and without appointment. Others attended the hospital specially. Normotensive subjects were recruited from accompanying relatives or friends and from hospital staff. There was some difficulty in recruiting subjects with DBP in the high range but, other than that, there were no problems. Patients on antihypertensive treatment attending the hospital were generally well controlled and therefore subjects in the high ranges had to be drawn mainly from new patients with some recruitment from those with renal disease.

\section{Screening and recruitment details}

\begin{tabular}{|c|c|c|c|c|c|c|c|}
\hline \multicolumn{3}{|l|}{ Screening and recruitment } & \multicolumn{5}{|c|}{ Recruitment ranges } \\
\hline Total screened & & 51 & & & $\mathrm{mmHg}$ & All & On Rx \\
\hline $\begin{array}{l}\text { Total excluded } \\
\text { Ranges complete }\end{array}$ & 11 & 18 & & Low & $\begin{array}{c}<90 \\
90-129\end{array}$ & $\begin{array}{r}0 \\
11\end{array}$ & 4 \\
\hline Range adjustment & 2 & & SBP & Medium & $130-160$ & 11 & 10 \\
\hline Arrhythmias & 1 & & & High & $161-180$ & 10 & 6 \\
\hline Device failure & 0 & & & Hign & $>180$ & 1 & 6 \\
\hline Poor quality sounds & 1 & & & & & & \\
\hline Cuff size unavailable & 0 & & & Low & $<40$ & 0 & 3 \\
\hline Observer disagreement & 0 & & & Low & $40-79$ & 11 & 3 \\
\hline Distribution & 2 & & DBP & Medium & $80-100$ & 12 & 9 \\
\hline Other reasons ${ }^{\mathrm{a}}$ & 1 & & & High & $101-130$ & 10 & 5 \\
\hline Total recruited & & 33 & & & $>130$ & 0 & \\
\hline
\end{tabular}

${ }^{a}$ Explanation summary. One subject had to leave for personal reasons before completing the sequence.

\section{Procedure}

The European Society of Hypertension International Protocol revision 2010 for the validation of blood pressure measuring devices in adults was followed precisely. [1] Overseen by an independent supervisor, measurements were recorded by two observers blinded from both each other's readings and from the device readings. 


\section{Results}

\section{Subject details}

Sex

Male : Female

Age (years)

Range (Low : High)

Mean (SD)

Arm circumference $(\mathrm{cm})$

Range (Low : High)

Mean (SD)

Cuff for test device

Standard

Large

Recruitment BP $(\mathrm{mmHg})$

Range (Low : High)

Mean (SD)

$$
\begin{aligned}
19 & : 14 \\
30 & : 72 \\
53.4 & (10.2) \\
22 & : 37 \\
29.8 & (2.9)
\end{aligned}
$$

$\begin{array}{cc}29 & (22-32 \mathrm{~cm}) \\ 4 & (33-43 \mathrm{~cm}) \\ \text { SBP } & \text { DBP }\end{array}$

$103: 178$

$146.3(25.0)$
$44: 123$

$90.1(16.8)$

Observer measurements in each recruitment range

\begin{tabular}{lccc}
\hline SBP $(\mathrm{mmHg})$ & \multicolumn{3}{c}{ DBP $(\mathrm{mmHg})$} \\
\hline Overall range $($ Low $:$ High $)$ & $103: 196$ & Overall range $($ Low $:$ High $)$ & $52: 135$ \\
Low $(<130)$ & 27 & Low $(<80)$ & 33 \\
Medium $(130-160)$ & 35 & Medium $(80-100)$ & 35 \\
High $(>160)$ & 37 & High $(>100)$ & 31 \\
Maximum difference & 10 & Maximum difference & 4 \\
\hline
\end{tabular}

Observer differences

\begin{tabular}{lccc}
\hline & SBP $(\mathrm{mmHg})$ & DBP $(\mathrm{mmHg})$ & Repeated measurements \\
\hline Observer 2-Observer 1 & & & \\
Range (Low : High) & $-4:+2$ & $-2:+3$ & 3 \\
Mean (SD) & $-1.1(1.2)$ & $+0.5(1.1)$ & 3 \\
\hline
\end{tabular}

Validation results

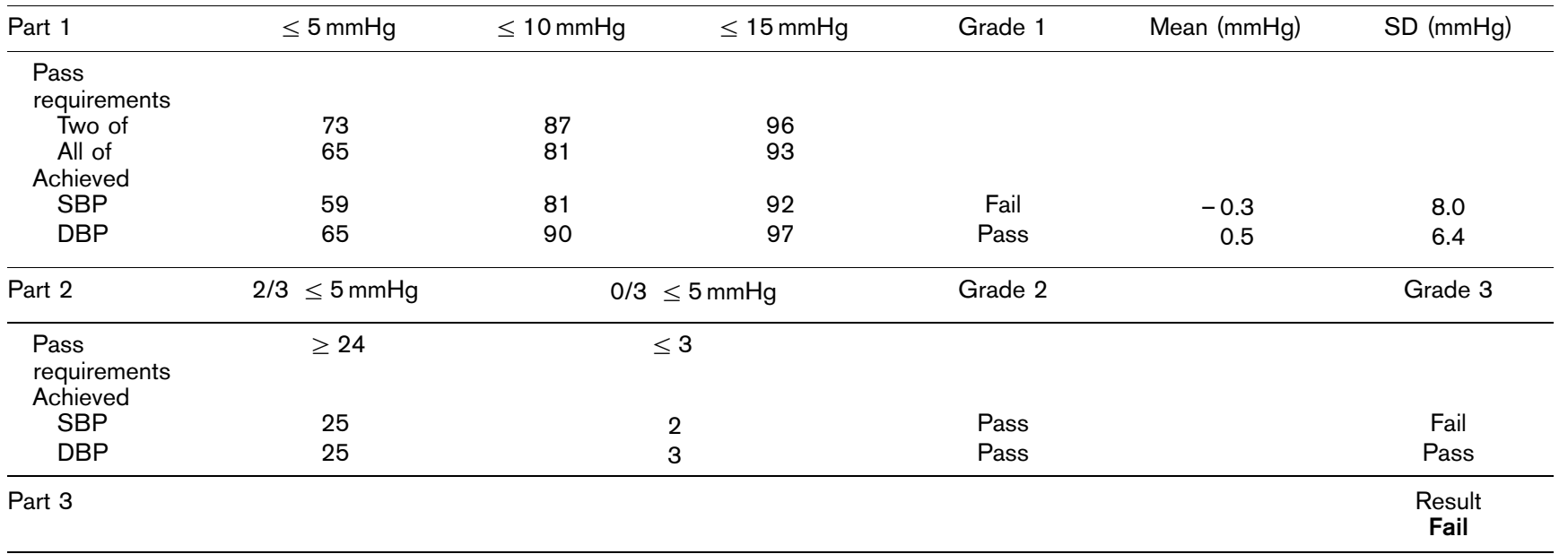




\section{Plots}
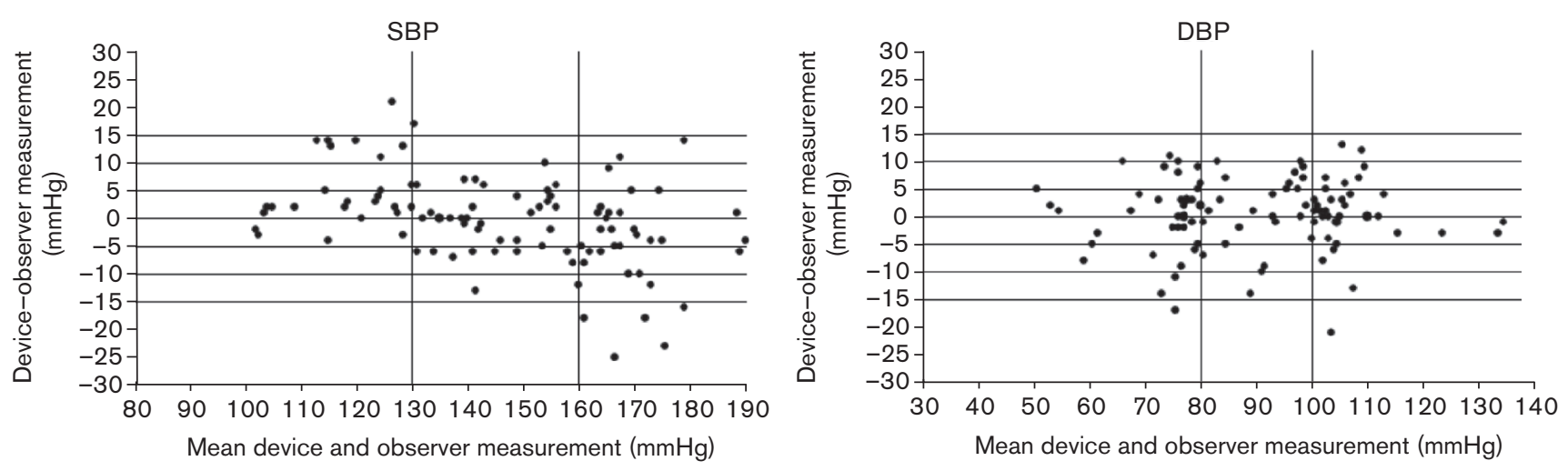

\section{Discussion}

Recruitment of subjects with high BP, in particular high DBP, proved to be extremely difficult and accounted for most of the extra screened subjects; this is reflected in the overall distribution, as shown in the DBP plot, in which most of the points are below $115 \mathrm{mmHg}$. Nevertheless, there is close to a perfect 33 points in each range and the distribution conditions are fulfilled. Therefore, this has had no effect on the result.

\section{Conclusion}

As the device has failed to reach the required standards, it is not recommended for personal or clinical use.

\section{Acknowledgements and conflicts of interest}

Two A1 monitors were supplied for the purposes of the study by the manufacturers Acme who also funded the study. None of the authors has any association with Acme or has received any personal benefit from Acme.

\section{References}

1 O'Brien E, Atkins N, Stergiou G, Karpettas N, Parati G, Asmar R, Imai Y, Wang J, Mengden T, Shennan A; on behalf of the Working Group on Blood Pressure Monitoring of the European Society of Hypertension. European Society of Hypertension International Protocol revision 2010 for the Validation of Blood Pressure Measuring Devices In Adults. Blood Press Monit 2010;15:23-38.

\section{Appendix B: Support facilities provided by the dabIEducational website to assist validation studies}

(1) The 2002 ESH International Protocol is available to download as a pdf file from www.dableducational.org [8]. This protocol outlines the history and development of validation protocol methodology.

(2) Manufacturers are referred to the device equivalence procedure on www.dableducational.org [18]. Manufacturers of blood pressure measuring devices may make modifications to a device, which has previously been successfully validated for accuracy, that do not affect its measurement accuracy. The modified device should not require further validation. The procedure for manufacturers to declare the equivalence of a modified device with a device that has been validated earlier is described.

(3) Manufacturers are referred to the facility for posting validation results on www.dableducational.org. This facility speeds up the posting of validation results on the dablEducational Trust website (without compromising later publication of a full paper).
(4) Manufacturers are referred to the facility for applying for performance accreditation for devices that is additional to the accuracy criteria of the International Protocol on www.dableducational.org and www.pressionearteriosa.net [19]. Although standard validation protocols provide assurance of the accuracy of blood pressure monitors, there is no guidance for the consumer as to the overall quality of a device. The PA.NET International Quality Certification Protocol denotes additional criteria of quality for blood pressure measuring devices. At the end of the certification process, ARSMED attributes a quality index to the device and a quality seal with four different grades (bronze, silver, gold and diamond) which may be used on the packaging of the appliance or in advertising. A quality certification is released to the manufacturer and published on www.pressionearteriosa. net and wwr.dableducational.org. 


\section{Appendix C: Membership of European Society of Hypertension working group on blood pressure monitoring}

Roland Asmar, Société Française d'Hypertension Artérielle, Filiale de la Société Française de Cardiolgie, 15, Rue de Cels-75014, Paris, France.

Lawrie Beilin, Department of medicine, University of Western Australia, Australia, GPO Box x2213, 35 Victoria Square, Perth WA 600, Australia.

Denis L. Clement, Afdeling Hart-en Vaatziekten, Universitair Ziekenhuis, De Pintelaan 185, B-9000 Gent, Belgium.

Peter De Leeuw, Interne Geneeskunde, Academisch Ziekenhuis, P. Debyelaan 25, postbus 5800, 6202 AZ Maastricht, The Netherlands.

Robert Fagard, Katholieke Universiteit Leuven, Hypertensie en Cardiovasculaire Inevalidatie Eenheid, Inwendige Geneeskunde-Cardiologie, U.Z. Gasthuisberg, Herestraat 49, 3000 Leuven, Belgium.

Yutaka Imai, The Department of Clinical Pharmacology and Therapeutics, Tohoku University Graduate School of Pharmaceutical Science and Medicine, 1-1 Seiryo-cho, Aoba-ku, Sendai 980-8574, Japan.

Jean-Michel Mallion, Médecine Interne et Cardiologie, Chef de Service, Centre Hospitalier Universitaire de Grenoble, B.P. 217 - 38043 Grenoble Cedex, France.

Giuseppe Mancia, Universita Degli Studi di Milano-Bicocca, Cattedra di Medicina Interna, Ospedale San Gerardo Dei Tintori, Via Donizetti 106, 20052 Monza, Italy.

Thomas Mengden, University Clinic Bonn, Department of Internal Medicine, Wilhelmstr. 35, 5311 Bonn, Germany.

Martin G. Myers, Division of Cardiology, Sunnybrook and Women's College Health Sciences Centre, 2075 Bayview Avenue, Toronto, Ontario M4N 3M5, Canada.

Eoin O'Brien, The Conway Institute of Biomolecular and Biomedical Research, University College Dublin, Belfield, Dublin 4, Ireland.

Paul Padfield, Department of Medicine, Western General Hospital, Edinburgh EH4 2XU, Scotland.
Gianfranco Parati, Istituto Scientifico Ospedale San Luca, IRCCS, Instituto Auxologico Italiano, Via Spagnoletto 3, 20149 Milan, Italy.

Paolo Palatini, Dipartimento di Medicina Clinica e Sperimentale, Universita' di Padova, Via Giustiniani 2, I-35128 Padua, Italy.

Josep Redon, Hypertension Clinic. Internal Medicine, Hospital Clinico, University of Valencia, Avda Blasco Ibañez 17, 46010 Valencia, Spain.

Jan Staessen (Chairman), Katholieke Universiteit Leuven, Hypertensie en Cardiovasculaire Revalidatie Eenheid, Inwendige Geneeskunde-Cardiologie, U.Z. Gasthuisberg, Herestraat 49, 3000 Leuven, Belgium.

George Stergiou, Hypertension Center, Third Department of Medicine, University of Athens, Sotiria Hospital, Athens, Greece.

Gert van Montfrans, Academisch Medisch Centrum, Interne Ziekten, Meibergdreef 9, AZ 1005 Amsterdam, The Netherlands.

Paolo Verdecchia, Departimento di discipline Cardiovascolari, Ospedale R. Silvestrini, Perugia, Italy.

Bernard Waeber (Secretary), Centre Hospitalier Universitaire Vaudois, Division D'Hypertension, Departement de medecine interne, 1011 Lausanne, Switzerland.

Jiguang Wang, Centre for Epidemiological Studies and Clinical Trials, Ruijin Hospital, Shanghai Jiaotong University School of Medicine, Ruijin 2nd Road 197, Shanghai 200025, China.

William White, Section of Hypertension and Vascular Diseases, The University of Connecticut Health Center, 263 Farmington Avenue, Farmington, Connecticut 060303940, USA. 